\title{
ON THE FLEXURAL AND EXTENSIONAL THERMOELASTIC WAVES IN ORTHOTROPIC PLATES WITH TWO THERMAL RELAXATION TIMES
}

\author{
K. L. VERMA AND NORIO HASEBE
}

Received 12 August 2003

Analysis for the propagation of plane harmonic thermoelastic waves in an infinite homogeneous orthotropic plate of finite thickness in the generalized theory of thermoelasticity with two thermal relaxation times is studied. The frequency equations corresponding to the extensional (symmetric) and flexural (antisymmetric) thermoelastic modes of vibration are obtained and discussed. Special cases of the frequency equations are also discussed. Numerical solution of the frequency equations for orthotropic plate is carried out, and the dispersion curves for the first six modes are presented for a representative orthotropic plate. The three motions, namely, longitudinal, transverse, and thermal, of the medium are found dispersive and coupled with each other due to the thermal and anisotropic effects. The phase velocity of the waves gets modified due to the thermal and anisotropic effects and is also influenced by the thermal relaxation time. Relevant results of previous investigations are deduced as special cases.

\section{Introduction}

The use of elastic waves to measure elastic properties as well as flaws of solid specimens has received interest, for example, in the use of elastic waves in nondestructive evaluation of concrete structures, in the use of laser-generated ultrasonic waves in the determination of anisotropic elastic constants of composite materials, and in the recovery of the bonding properties and/or thickness of bonded structures. The growing applications of new composite materials, especially in thermal environment, have encouraged the studies of impact and wave propagation in the composite materials and have become very important. The theory to include the effect of temperature change, known as the theory of thermoelasticity, is well established $[5,19,20]$. Classical theory of dynamic thermoelasticity that takes into account the coupling effects between temperature and strain fields involves the infinite thermal wave speed, that is, it implies an immediate response to a temperature gradient and leads to a parabolic differential equation for the evolution of the temperature. In contrast, when relaxation effects are taken into account in the constitutive equation describing the heat flux, as, for instance, in the Maxwell-Cattaneo equation, one has a hyperbolic equation which implies a finite speed for heat transport. 
Hyperbolic heat transport has been receiving increasing attention both for theoretical motivations (analysis of thermal waves and second sound in dielectric solids, finite speed of heat transport, etc.) and for the analysis of some practical problems involving a fast supply of thermal energy (e.g., by a laser pulse or a chemical explosion, etc.). The usual theory of thermal conduction, based on the Fourier law, implies an immediate response to a temperature gradient and leads to a parabolic differential equation for the evolution of the temperature. In contrast, when relaxation effects are taken into account in the constitutive equation describing the heat flux, heat conduction equation becomes a hyperbolic equation, which implies a finite speed for heat transport. Waves' types occurring in bounded anisotropic media are very complicated, and in thermoelasticity, the problem becomes even more complicated because solutions to both the heat conduction and thermoelasticity problems for anisotropy are required. These solutions are also to satisfy the thermal and mechanical boundary and interface conditions. The literature dedicated to such theories (hyperbolic thermoelastic models) is quite large and its detailed review can be found in Chandrasekharaiah $[8,9]$.

Lord and Shulman [14] and Green and Lindsay [11], extended the coupled theory of thermoelasticity by introducing the thermal relaxation time in the constitutive equations. This new theory, which eliminates the paradox of infinite velocity of heat propagation, is called generalized theory of thermoelasticity. This generalized thermoelasticity theory that admits finite speed for the propagation of thermoelastic disturbances has received much attention in recent years. The LS model introduces a single time constant to dictate the relaxation of thermal propagation as well as the rate of change of strain rate and the rate of change of heat generation. In the GL theory, on the other hand, the thermal and thermomechanical relaxations are governed by two different time constants.

The propagation of thermoelastic waves in a plate under plane stress by using generalized theories of thermoelasticity has been studied by Massalas [15]. Here, we mention that several authors (see $[3,2,16,17,21,22,23]$ ) have considered the propagation of generalized thermoelastic waves in plates of isotropic media. Propagation of generalized thermoelastic vibrations in infinite plates in the context of generalized thermoelasticity is studied [25].

The thermoelastic wave propagation in transversely isotropic and homogeneous anisotropic heat-conducting elastic materials is investigated in $[6,7]$, respectively. This theory extended to anisotropic heat conducting elastic materials in $[4,10]$ treated the problem in a more systematic manner. They derived governing field equations of generalized thermoelastic media and proved that these equations are unique. A thermoelastic problem is studied in [24], considering equations for anisotropic heat conducting solids with thermal relaxation time. Hawwa and Nayfeh [12] studied the general problem of thermoelastic waves in anisotropic periodically laminated composites. In [26, 27], wave propagation in plates of general anisotropic media for generalized thermoelasticity is studied.

In this paper, the problem of plane harmonic thermoelastic waves in an infinite homogeneous orthotropic plate of finite thickness in the generalized theory of thermoelasticity with two thermal relaxation times is studied. The results obtained theoretically have been verified numerically and represented graphically for a representative orthotropic plate. Longitudinal, transverse, and thermal motions of the medium are found coupled with 
each other and are dispersive. It is also shown that phase velocity of the waves is influenced by the thermal relaxation times. Special cases have also been discussed.

\section{Formulation}

Consider a set of Cartesian coordinate system $x_{i}=\left(x_{1}, x_{2}, x_{3}\right)$ in such a manner that the $x_{3}$ axis is normal to the layering. The basic field equations of generalized thermoelasticity for an infinite generally anisotropic thermoelastic medium at uniform temperature $T_{0}$ in the absence of body forces and heat sources [23] are

$$
\sigma_{i j, j}=\rho \ddot{u}_{, i} \quad K_{i j} T_{, i j}-\rho C_{e}\left(\dot{T}+\tau_{0} \ddot{T}\right)=T_{0} \beta_{i j} \dot{u}_{i, j},
$$

where

$$
\begin{aligned}
& \sigma_{i j}=c_{i j k l} e_{k l}-\beta_{i j}\left(T+\tau_{1} \dot{T}\right), \\
& \beta_{i j}=c_{i j k l} \alpha_{k l}, \quad i, j, k, l=1,2,3,
\end{aligned}
$$

$\rho$ is the density, $t$ is the time, $u_{i}$ is the displacement in the $x_{i}$ direction, $K_{i j}$ are the thermal conductivities, $C_{e}$ and $\tau_{0}$ are, respectively, the specific heat at constant strain and thermal relaxation time, $\sigma_{i j}$ and $e_{i j}$ are the stress and strain tensor, respectively, $\beta_{i j}$ are thermal moduli, $\alpha_{i j}$ is the thermal expansion tensor, $T$ is the temperature, and the fourth-order tensor of the elasticity $C_{i j k l}$ satisfies the (Green) symmetry conditions:

$$
c_{i j k l}=c_{k l i j}=c_{i j l k}=c_{j i k l}, \quad \alpha_{i j}=\alpha_{j i}, \quad \beta_{i j}=\beta_{j i} .
$$

The parameters $\tau_{1}$ and $\tau_{0}$ are the thermal-mechanical relaxation time and the thermal relaxation time of the GL theory, and they satisfy the inequality $\tau_{1} \geq \tau_{0} \geq 0$. Comma notation is used for spatial derivatives, and superposed dot represents differentiation with respect to time.

We have the strain-displacement relation

$$
e_{i j}=\frac{\left(u_{i, j}+u_{j, i}\right)}{2}
$$

The stresses, temperature gradient, displacements, and the temperature components at the surface of the plate are

$$
S\left(x_{3}\right)=\left(\bar{\sigma}_{13}, \bar{\sigma}_{23}, \bar{\sigma}_{33}, \frac{\partial \bar{T}}{\partial x_{3}}\right), \quad D\left(x_{3}\right)=\left(\bar{u}_{1}, \bar{u}_{2}, \bar{u}_{3}, \bar{T}\right)
$$

and the bar means the amplitudes of the displacement; temperature, stress, and the temperature gradient are functions of $x_{3}$ only.

The boundary conditions on the plate surfaces are

$$
\begin{array}{r}
S(-d)=\mathbf{0}, \\
S(d)=\mathbf{0},
\end{array}
$$

where $\mathbf{0}$ is a zero vector. 
72 Flexural and extensional thermoelastic waves

When specializing (2.1), (2.2), and (2.3) for orthotropic media in generalized thermoelasticity, the governing equations are

$$
\begin{gathered}
\left(c_{11} u_{1,11}+c_{66} u_{1,22}+c_{55} u_{1,33}\right)+\left(c_{12}+c_{66}\right) u_{2,12}+\left(c_{13}+c_{55}\right) u_{3,13}-\beta_{1}\left(T+\tau_{1} \dot{T}\right)_{, 1}=\rho \ddot{u}_{1}, \\
\left(c_{12}+c_{66}\right) u_{1,12}+\left(c_{66} u_{2,11}+c_{22} u_{2,22}+c_{44} u_{2,33}\right)+\left(c_{23}+c_{44}\right) u_{3,23}-\beta_{2}\left(T+\tau_{1} \dot{T}\right)_{, 2}=\rho \ddot{u}_{2}, \\
\left(c_{13}+c_{55}\right) u_{1,13}+\left(c_{23}+c_{44}\right) u_{2,23}+c_{55} u_{3,11}+c_{44} u_{3,22}+c_{33} u_{3,33}-\beta_{3}\left(T+\tau_{1} \dot{T}\right)_{, 3}=\rho \ddot{u}_{3}, \\
\left(K_{11} T_{, 11}+K_{22} T_{, 22}+K_{33} T_{, 33}\right)-\rho C_{e}\left(\dot{T}+\tau_{0} \ddot{T}\right)=T_{0}\left[\beta_{1} \dot{u}_{1,1}+\beta_{2} \dot{u}_{2,2}+\beta_{3} \dot{u}_{3,3}\right],
\end{gathered}
$$

where

$$
\begin{aligned}
& \beta_{1}=c_{11} \alpha_{1}+c_{12} \alpha_{2}+c_{13} \alpha_{3}, \\
& \beta_{2}=c_{12} \alpha_{1}+c_{22} \alpha_{2}+c_{23} \alpha_{3}, \\
& \beta_{3}=c_{13} \alpha_{1}+c_{32} \alpha_{2}+c_{33} \alpha_{3} .
\end{aligned}
$$

\section{Solution}

Having identified the plane of incidence to be the $x_{1}-x_{3}$ plane, then the solution for displacements and temperature for an angle of incidence $\theta$ is proposed:

$$
\left(u_{j}, T\right)=\left(U_{j}, U_{4}\right) \exp \left[i \xi\left(\sin \theta x_{1}+\alpha x_{3}-c t\right)\right], \quad i=\sqrt{-1}, j=1,2,3,
$$

where $\xi$ is the wave number, $c$ is the phase velocity $(=\omega / \xi), \omega$ is the circular frequency, $\alpha$ is still an unknown parameter, $U_{j}$ and $U_{4}$ are the constants related to the amplitudes of displacement $u_{1}, u_{2}, u_{3}$, and the temperature T. Although solution (3.1) is explicitly independent of $x_{2}$, an implicit dependence is contained in the transformation, and the transverse displacement component $u_{2}$ is nonvanishing in (3.1).

Substituting (3.1) in (2.8) leads to the coupled equations, the choice of solutions leads to four coupled equations:

$$
M_{m n}(\alpha) U_{n}=\mathbf{0}, \quad m, n=1,2,3,4,
$$

where

$$
\begin{gathered}
M_{11}=F_{11}+c_{2} \alpha^{2}, \quad M_{13}=F_{13} \alpha, \quad M_{14}=F_{14}, \\
M_{22}=F_{22}+c_{6} \alpha^{2}, \quad M_{24}=F_{24}, \\
M_{33}=F_{33}+c_{1} \alpha^{2}, \quad M_{34}=F_{34} \alpha, \\
M_{41}=F_{41}, \quad M_{43}=F_{43} \alpha, \quad M_{44}=F_{44}+\bar{K} \alpha^{2},
\end{gathered}
$$

where

$$
\begin{gathered}
F_{11}=\sin ^{2} \theta-\zeta^{2}, \quad F_{13}=c_{7} \sin \theta, \quad F_{14}=\sin \theta, \\
F_{22}=c_{3} \sin ^{2} \theta-\zeta^{2}, \quad F_{33}=c_{2} \sin ^{2} \theta-\zeta^{2}, \quad F_{34}=\bar{\beta}_{3} \alpha, \\
F_{41}=\varepsilon_{1} \tau_{g} \omega_{1}^{*} \zeta^{2} \sin \theta, \quad F_{43}=\varepsilon_{1} \tau_{g} \omega_{1}^{*} \zeta^{2} \bar{\beta}_{1} \alpha, \quad F_{44}=\sin ^{2} \theta-\tau \omega_{1}^{*} \zeta^{2},
\end{gathered}
$$


and

$$
\begin{gathered}
c_{1}=\frac{c_{33}}{c_{11}}, \quad c_{2}=\frac{c_{55}}{c_{11}}, \quad c_{3}=\frac{c_{66}}{c_{11}}, \quad c_{6}=\frac{c_{44}}{c_{11}}, \quad c_{7}=\frac{c_{13}+c_{55}}{c_{11}}, \\
\bar{\beta}_{3}=\frac{\beta_{3}}{\beta_{1}}, \quad \bar{K}=\frac{K_{3}}{K_{1}}, \quad \varepsilon_{1}=\frac{T_{0} \beta_{1}^{2}}{\rho C_{e} c_{11}}, \quad \omega_{1}^{*}=\frac{C_{e} C_{11}}{K_{1}}, \\
\zeta^{2}=\frac{c^{2} \rho}{c_{11}}, \quad \tau=i \omega^{-1}+\tau_{0}, \quad \tau_{g}=i \omega^{-1}+\tau_{1} .
\end{gathered}
$$

The system of (3.2) has a nontrivial solution if the determinant of the coefficients of $U_{1}$, $U_{2}, U_{3}$, and $U_{4}$ vanishes, which yields an algebraic equation relating $\alpha$ to $c$. We obtain a polynomial equation in $\alpha$, which can be written as

$$
\begin{gathered}
\alpha^{6}+A_{1} \alpha^{4}+A_{2} \alpha^{2}+A_{3}=0, \\
c_{3}+c_{6} \alpha^{2}-\zeta^{2}=0,
\end{gathered}
$$

where

$$
\begin{aligned}
& A_{1}=\frac{\left[P(-\bar{K})+\Delta_{1} F_{44}-c_{2} F_{34} F_{43}\right]}{\Delta}, \\
& A_{2}=\frac{\left[Q(-\bar{K})+P F_{44}+\left(F_{13} F_{34}-c_{1} F_{14}\right) F_{41}+\left(F_{13} F_{14}-F_{11} F_{34}\right) F_{43}\right]}{\Delta}, \\
& A_{3}=\frac{\left(R F_{44}-F_{14} F_{33} F_{41}\right)}{\Delta},
\end{aligned}
$$

where

$$
\begin{aligned}
P= & \left(c_{1} c_{6} F_{11}-c_{6} F_{13}^{2}+c_{5} F_{13} F_{23}-c_{5}^{2} F_{33}\right. \\
& \left.-c_{2} F_{23}^{2}-2 c_{1} c_{5} F_{12}+c_{5} F_{21} F_{23}+c_{2} c_{6} F_{33}\right), \\
Q= & \left(F_{11} F_{23}^{2}-F_{13}^{2} F_{22}+c_{6} F_{11} F_{33}+c_{1} F_{11} F_{22}\right. \\
& \left.-c_{1} F_{12}^{2}+2 F_{12} F_{13} F_{23}+2 c_{5} F_{12} F_{33}+c_{2} F_{22} F_{33}\right), \\
R= & \left(F_{11} F_{22}-F_{12}^{2}\right) F_{33}, \\
\Delta= & -\bar{K}\left(c_{2} c_{6}-c_{5}^{2}\right) c_{1}, \\
\Delta_{1}= & \left(c_{2} c_{6}-c_{5}^{2}\right) c_{1} .
\end{aligned}
$$

Notice that roots of (3.7) corresponding to the SH motion give a purely transverse wave, which is not affected by the temperature. This wave propagates without dispersion or damping. 
74 Flexural and extensional thermoelastic waves

Equation (3.6) corresponds to the sagittal plane waves, and for the motion in this plane, each $\alpha_{l}, l=1,2, \ldots, 6$ the displacements, temperature, stress, and temperature gradient amplitudes are

$$
\begin{gathered}
q_{3(l)}=\frac{\left[F_{44}\left(F_{11}+c_{2} \alpha_{l}^{2}\right)-F_{14} F_{41}-\left(c_{2} \alpha_{l}^{4}+F_{11} \alpha_{l}^{2}\right) \bar{K}\right]}{\left[F_{14} F_{43} \alpha_{l}-F_{13} F_{44}+\bar{K} F_{13} \alpha_{l}^{2}\right] \alpha_{l}}, \\
\Theta_{l}=\frac{\left[F_{13} F_{41}-\left(F_{11}+c_{2} \alpha_{l}^{2}\right) F_{43}\right]}{\left[F_{14} F_{43}-F_{13}\left(F_{44}-\bar{K} \alpha_{l}^{2}\right)\right]}, \\
r_{33(l)}=\left[i \xi\left\{\left(c_{7}-c_{2}\right) \sin \theta+c_{1} \alpha_{l} q_{3(l)}\right\}+i \xi^{-1} \beta_{3} c_{11}^{-1}\left(1-c \tau_{1}\right) \Theta_{l}\right], \\
r_{13(l)}=i \xi\left[c_{2}\left(\alpha_{l}+q_{3(l)} \sin \theta\right)\right], \\
\Omega_{l}=\iota \xi \alpha_{l} \Theta_{l} .
\end{gathered}
$$

For the SH-type wave, one now has

$$
r_{23(8)}=-r_{23(7)}=c_{6} \alpha_{7}
$$

As (3.10) admits solutions for $\alpha$, having the properties $\alpha_{2 l}=-\alpha_{2 l-1}, l=1,2,3$, incorporating this property into (3.10) and (3.11), we have

$$
q_{3(2 l)}=-q_{3(2 l-1)}, \quad \Theta_{2 l}=\Theta_{2 l-1} .
$$

\section{Dispersion relation}

If the roots of bicubic equation (3.6) are denoted by $\alpha_{1}^{2}, \alpha_{2}^{2}$, and $\alpha_{3}^{2}$, then solutions of $u_{1}, u_{3}$, and $T$ are then being obtainable as linear combinations of six linear independent solutions corresponding to $\alpha_{l}, l=1,2, \ldots, 6$, with property $\alpha_{2 l-1}=-\alpha_{l}, l=1,2,3$. The equations of motion and heat conduction may be used to establish the formal solution for the displacement and temperature as

$$
\left(u_{1}, u_{3}, T\right)=\sum_{l=1}^{6}\left(1, q_{3(l)}, \Theta_{l}\right) A_{l} \exp \left(\iota \xi \alpha_{l} x_{3}\right) \exp \left[\iota \xi\left(x_{1} \sin (\theta)-c t\right)\right] .
$$

As (3.6) admits solutions for $\alpha$, having the properties $\alpha_{2 l-1}=-\alpha_{l}, l=1,2,3$, we therefore have $\left(u_{1}, u_{3}, T\right)=\left(\bar{u}_{1}, \bar{u}_{3}, \bar{T}\right) \exp \left[\iota \xi\left(x_{1} \sin (\theta)-c t\right)\right]$, where

$$
\begin{aligned}
& \bar{u}_{1}=\sum_{l=1}^{3}\left(U^{(2 l-1)} E_{l}^{+}+U^{(2 l)} E_{l}^{-}\right), \\
& \bar{u}_{3}=\sum_{l=1}^{3} q_{3(l)}\left(U^{(2 l-1)} E_{l}^{+}-U^{(2 l)} E_{l}^{-}\right), \\
& \bar{T}=\sum_{l=1}^{3} \Theta_{l}\left(U^{(2 l-1)} E_{l}^{+}+U^{(2 l)} E_{l}^{-}\right),
\end{aligned}
$$


where

$$
E_{l}^{+}=e^{i \xi \alpha_{l} d}, \quad E_{l}^{-1}=e^{-i \xi \alpha_{l} d}, \quad l=1,2,3,
$$

and $U^{(i)}, i=1,2, \ldots, 6$, are disposal constants. The disposal constants for $U^{(i)}$, are not independent as they are linked through the equations of motion and heat conduction. Here, $q_{3(l)}$ are the displacements ratios, and $\Theta_{l}$ the temperature of displacement ratios defined in (3.10) and (3.11).

Combining (4.1), (4.2), (3.10), and (3.11) with the stress-strain and temperature relations, and using superposition, we write stresses and temperature gradient as

$$
\left(\sigma_{33}, \sigma_{13}, T^{\prime}\right)=\left(\bar{\sigma}_{33}, \bar{\sigma}_{13}, \overline{T^{\prime}}\right) \exp \left[\iota \xi\left(x_{1}+\alpha x_{2}-c t\right)\right]
$$

with

$$
\begin{aligned}
& \bar{\sigma}_{33}=\sum_{l=1}^{3} r_{33(l)}\left(U^{(2 l-1)} E_{l}^{+}+U^{(2 l)} E_{l}^{-}\right), \\
& \bar{\sigma}_{13}=\sum_{l=1}^{3} r_{13(l)}\left(U^{(2 l-1)} E_{l}^{+}+U^{(2 l)} E_{l}^{-}\right), \\
& \overline{T^{\prime}}=\sum_{l=1}^{3} \Omega_{l}\left(U^{(2 l-1)} E_{l}^{+}+U^{(2 l)} E_{l}^{-}\right),
\end{aligned}
$$

where $r_{33(l)}, r_{13(l)}$, and $\Omega_{l}, l=1,2,3, \ldots, 6$, are defined in (3.12), (3.13), and (3.14).

As (3.10) admits solutions for $\alpha$, having the properties $\alpha_{2 l-1}=-\alpha_{l}$, incorporating this property into (3.10), (3.11), (3.12), (3.13), and (3.14) and inspecting the resulting relations, we conclude the further restrictions

$$
\begin{gathered}
r_{33(2 l)}=r_{33(2 l-1)}, \\
r_{13(2 l)}=-r_{13(2 l-1)}, \\
\Omega_{2 l}=-\Omega_{2 l-1}, \quad l=1,3,5 .
\end{gathered}
$$

The dispersion relation associated with the plate is now derived from (4.4) by applying traction-free and thermally insulated boundaries boundary conditions (2.6) and (2.7) at the upper and lower faces $x_{3}= \pm d$ of the plate, thus

$$
\begin{aligned}
& \sum_{l=1}^{3} r_{33(l)}\left(U^{(2 l-1)} e^{i \xi \alpha_{l} d}+U^{(2 l)} e^{-i \xi \alpha_{l} d}\right)=0, \\
& \sum_{l=1}^{3} r_{33(l)}\left(U^{(2 l-1)} e^{-i \xi \alpha_{l} d}+U^{(2 l)} e^{i \xi \alpha_{l} d}\right)=0 \\
& \sum_{l=1}^{3} r_{13(l)}\left(U^{(2 l-1)} e^{i \xi \alpha_{l} d}-U^{(2 l)} e^{-i \xi \alpha_{l} d}\right)=0
\end{aligned}
$$


76 Flexural and extensional thermoelastic waves

$$
\begin{aligned}
& \sum_{l=1}^{3} r_{13(l)}\left(U^{(2 l-1)} e^{-i \xi \alpha_{l} d}-U^{(2 l)} e^{i \xi \alpha_{l} d}\right)=0 \\
& \sum_{l=1}^{3} \Omega_{(l)}\left(U^{(2 l-1)} e^{i \xi \alpha_{l} d}-U^{(2 l)} e^{-i \xi \alpha_{l} d}\right)=0 \\
& \sum_{l=1}^{3} \Omega_{(l)}\left(U^{(2 l-1)} e^{-i \xi \alpha_{l} d}-U^{(2 l)} e^{i \xi \alpha_{l} d}\right)=0 .
\end{aligned}
$$

On further simplifying equations (4.7), we have

$$
\begin{aligned}
\sum_{l=1}^{3} r_{33(l)}\left(\tilde{U}_{l}^{+} C_{l}+i \tilde{U}_{l}^{-} S_{l}\right) & =0, & & \sum_{l=1}^{3} r_{33(l)}\left(\tilde{U}_{l}^{+} C_{l}-i \tilde{U}_{l}^{-} S_{l}\right)=0, \\
\sum_{l=1}^{3} r_{13(l)}\left(\tilde{U}_{l}^{-} C_{l}+i \tilde{U}_{l}^{+} S_{l}\right) & =0, & & \sum_{l=1}^{3} r_{13(l)}\left(\tilde{U}_{l}^{-} C_{l}-i \tilde{U}_{l}^{+} S_{l}\right)=0, \\
\sum_{l=1}^{3} \Omega_{l}\left(\tilde{U}_{l}^{-} C_{l}+i \tilde{U}_{l}^{+} S_{l}\right) & =0, & & \sum_{l=1}^{3} \Omega_{l}\left(\tilde{U}_{l}^{-} C_{l}-i \tilde{U}_{l}^{-} S_{l}\right)=0 .
\end{aligned}
$$

The symmetry of the plate allows us to simplify the system of six homogeneous equations in six unknowns into two systems of three equations in three unknowns, which on employing straightforward algebraic manipulations yield the following relations associated with the plate:

$$
\begin{gathered}
\sum_{l=1}^{3} r_{33(l)} \tilde{U}_{l}^{+} C_{l}=0, \\
\sum_{l=1}^{3} r_{13(l)} \tilde{U}_{l}^{+} S_{l}=0, \\
\sum_{l=1}^{3} \Omega_{l} \tilde{U}_{l}^{+} S_{l}=0,
\end{gathered}
$$

and

$$
\begin{gathered}
\sum_{l=1}^{3} r_{33(l)} \tilde{U}_{l}^{-} S_{l}=0, \\
\sum_{l=1}^{3} r_{13(l)} \tilde{U}_{l}^{-} C_{l}=0, \\
\sum_{l=1}^{3} \Omega_{l} \tilde{U}_{l}^{-} C_{l}=0,
\end{gathered}
$$


within which

$$
\begin{array}{cl}
C_{l}=\cos \left(\xi \alpha_{l} d\right), & S_{l}=\sin \left(\xi \alpha_{l} d\right), \\
\tilde{U}_{l}^{+}=U^{(2 l-1)}+U^{(2 l)}, & \tilde{U}_{l}^{-}=U^{(2 l-1)}-U^{(2 l) .}
\end{array}
$$

The condition that the systems of (4.9), (4.10), and (4.11), and (4.12), (4.13), and (4.14) admit a nontrivial solution gives rise to the dispersion relations associated with extensional and flexural waves, respectively.

\section{Flexural waves}

The dispersion relation associated with flexural waves equation is obtained by taking $U^{(2 l-1)}=U^{(2 l)}$, thus, $\bar{u}_{1}, \bar{u}_{3}$, and $\bar{T}$ have the form

$$
\bar{u}_{1}=2 \sum_{l=1}^{3} U^{(2 l)} C_{l}, \quad \bar{u}_{3}=2 i \sum_{l=1}^{3} q_{3(l)} U^{(2 l)} S_{l}, \quad \bar{T}=2 \sum_{l=1}^{3} \Theta_{l} U^{(2 l)} C_{l},
$$

and therefore require that the system of (4.12), (4.13), and (4.14) admit a nontrivial solution provided that the determinant of coefficients associated with these equations vanishes, which after a little and straightforward algebraic manipulation, may cast in the form

$$
r_{33(1)} G_{1} \Gamma_{1}+r_{33(2)} G_{2} \Gamma_{2}+r_{33(3)} G_{3} \Gamma_{3}=0,
$$

where

$$
\begin{gathered}
G_{1}=r_{13(2)} \Theta_{3}-r_{13(3)} \Theta_{2}, \quad G_{2}=r_{13(3)} \Theta_{1}-r_{13(1)} \Theta_{3}, \\
G_{3}=r_{13(1)} \Theta_{2}-r_{13(2)} \Theta_{1} \\
\Gamma_{l}=\tan \left(\gamma \alpha_{l}\right), \quad \gamma=\xi d=\frac{\omega}{c} .
\end{gathered}
$$

\section{Extensional waves}

The dispersion relation associated with the extensional waves equation is obtained by taking $U^{(2 l-1)}=-U^{(2 l)}$, and the determinant of the coefficients of (4.9), (4.10), and (4.11) yields the dispersion relation associated with extensional waves, namely,

$$
r_{33(1)} G_{1} \Gamma_{2} \Gamma_{3}+r_{33(2)} G_{2} \Gamma_{1} \Gamma_{3}+r_{33(3)} G_{3} \Gamma_{1} \Gamma_{2}=0,
$$

thus, $\bar{u}_{1}, \bar{u}_{3}$, and $\bar{T}$ have the form

$$
\bar{u}_{1}=-2 i \sum_{l=1}^{3} U^{(2 l)} S_{l}, \quad \bar{u}_{3}=-2 \sum_{l=1}^{3} q_{3(l)} U^{(2 l)} C_{l}, \quad \bar{T}=-2 i \sum_{l=1}^{3} \Theta_{l} U^{(2 l)} S_{l},
$$

$G_{1}, G_{2}, G_{3}$, and $\Gamma_{l}$ are defined in (5.3). 


\section{Special cases}

7.1. Classical case. If $\varepsilon_{1}=0$, then thermal and elastic fields decoupled from each other and from (3.6) become the characteristic equation in the uncoupled thermoelasticity.

We have

$$
M_{41}=M_{43}=0,
$$

and (3.6) reduces to

$$
\left(B_{1} \alpha^{4}+B_{2} \alpha^{2}+B_{3}\right) M_{44}(\alpha)=0
$$

where

$$
M_{44}=1-\tau \omega_{1}^{*} \zeta^{2}+\bar{K} \alpha^{2}=0
$$

and $B_{1} \alpha^{4}+B_{2} \alpha^{2}+B_{3}=0$ is a secular equation corresponding to the purely elastic material, which is obtained and discussed in $[1,18]$.

Equation (7.3) provides

$$
1-\tau \omega_{1}^{*} \zeta^{2}+\bar{K} \alpha^{2}=0
$$

which corresponds to the thermal wave. Clearly it is influenced by the thermal relaxation time $\tau_{0}$ in the Green-Lindsay theory.

7.2. Coupled thermoelasticity. This case corresponds to no thermal relaxation time, that is, $\tau_{0}=\tau_{1}=0$ and hence $\tau=\tau_{g}=i / \omega$. In case, proceeding on the same lines, we again arrive at frequency equations of the form that is again in agreement with the corresponding result obtained in $[3,13,24]$.

If $\tau_{1}=\tau_{0} \neq 0,(5.2)$ and (6.1) become the frequency equations in the LS theory of generalized thermoelasticity (see [24]).

7.3. Cubic and isotropic materials. Results for materials possessing transverse isotropy, cubic symmetry, and isotropic case, can be easily obtained from (5.2) and (6.1) by imposing the additional conditions on the thermoelastic constants, namely,

$$
\begin{gathered}
c_{33}=c_{22}, \quad \begin{array}{c}
c_{13}=c_{12}, \quad c_{55}=c_{66}, \quad c_{22}-c_{23}=2 c_{44}, \\
K_{1}=K_{2}, K_{3}, \quad \alpha_{1}=\alpha_{2}, \alpha_{3},
\end{array} \\
\beta_{1}=\beta_{2}=\left(c_{11}+c_{12}\right) \alpha_{1}+c_{13} \alpha_{3}, \quad \beta_{3}=2 c_{13} \alpha_{1}+c_{33} \alpha_{3},
\end{gathered}
$$

and for cubic symmetry,

$$
\begin{aligned}
& c_{11}=c_{22}=c_{33}, \quad c_{13}=c_{12}=c_{23}, \quad c_{44}=c_{55}=c_{66}, \\
& K_{1}=K_{2}=K_{3}, \quad \alpha_{1}=\alpha_{2}=\alpha_{3}=\alpha_{t}, \quad \beta_{1}=\beta_{2}=\beta_{3}=\beta=\left(c_{11}+c_{12}\right) \alpha_{t} \text {. }
\end{aligned}
$$




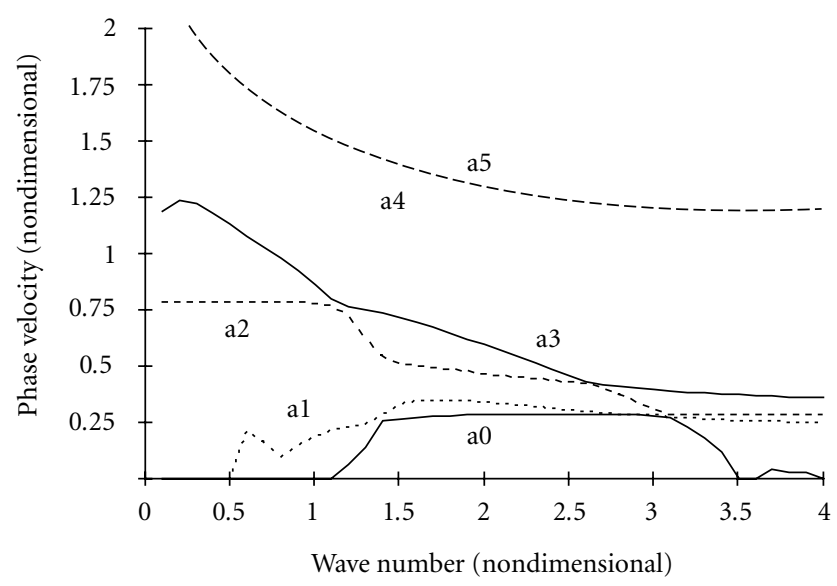

Figure 8.1. Dispersion of the first six flexural modes for $\tau_{0}=2 \cdot 10^{-7}$ second and $\tau_{1}=4 \cdot 10^{-7}$ second.

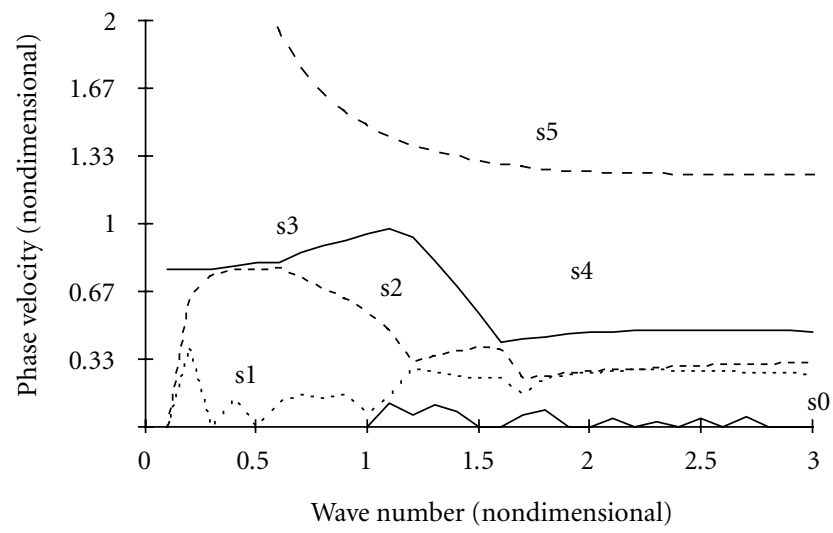

Figure 8.2. Dispersion of the first six extensional modes for $\tau_{0}=2.10^{-7}$ second and $\tau_{1}=$ $4.10^{-7}$ second.

Finally, for the isotropic case,

$$
\begin{aligned}
& c_{11}=c_{22}=c_{33}=\lambda+2 \mu, \quad c_{13}=c_{12}=c_{23}=\lambda, \quad c_{44}=c_{55}=c_{66}=\mu, \\
& K_{1}=K_{2}=K_{3}, \quad \alpha_{1}=\alpha_{2}=\alpha_{3}=\alpha_{t}, \quad \beta_{1}=\beta_{2}=\beta_{3}=(3 \lambda+2 \mu) \alpha_{t} .
\end{aligned}
$$

\section{Numerical results and discussion}

Numerical illustrations of the analytical characteristic equations are presented in the form of dispersion curves. These curves are obtained by keeping $\xi$ (wave number) real and letting $c$ be complex. Then the phase velocity is defined as $\operatorname{Re}(c)$, and the imaginary part of $c$ is a measure of the damping of the waves. One can also let $c$ be real and let $\xi$ be complex. In this case, the wave $c$ corresponding to $\operatorname{Re}(\xi)$ and $\operatorname{Im}(\xi)$ is a measure of the attenuation of the wave. To find the solutions of a characteristic equation, Mathcad software is used 


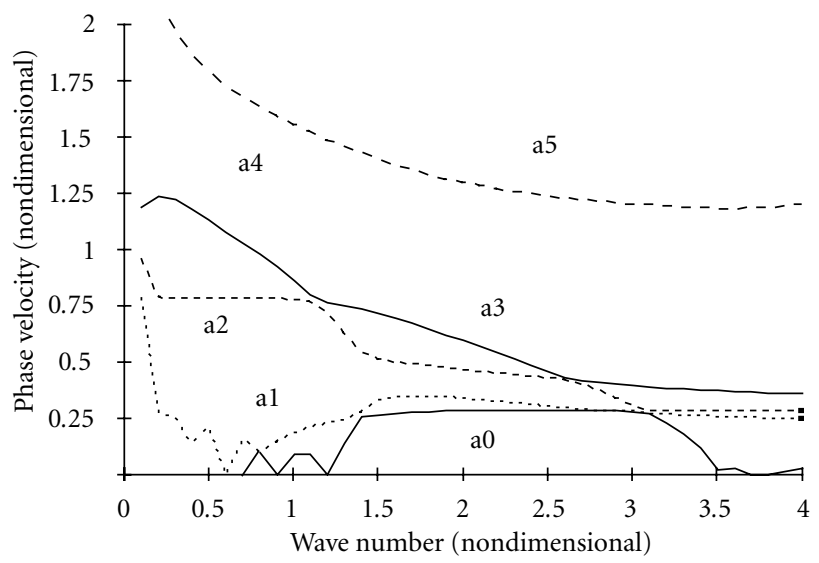

Figure 8.3. Dispersion of the first six flexural modes for $\tau_{0}=2.10^{-7}$ second and $\tau_{1}=1.10^{-6}$ second.

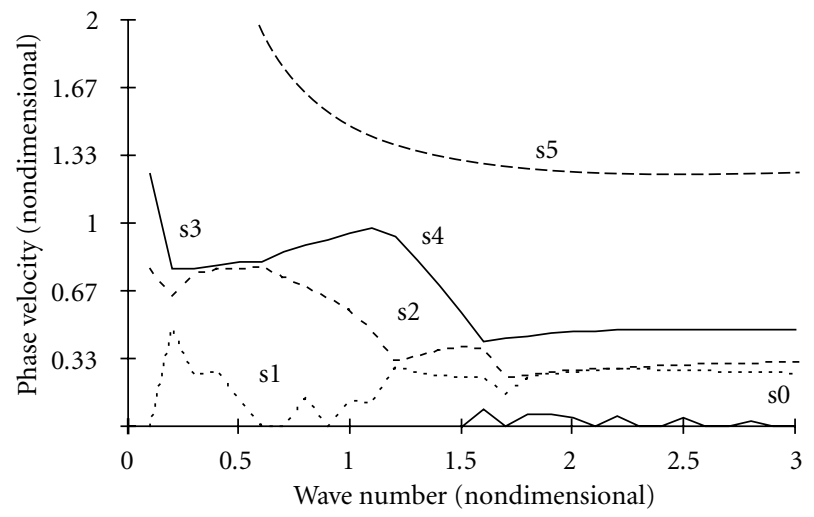

Figure 8.4. Dispersion of the first six extensional modes for $\tau_{0}=2.10^{-7}$ second and $\tau_{1}=1.10^{-6}$ second.

to solve it as an analytic function by considering representative orthotropic (fictitious) material given in [12] with the following properties:

$$
\begin{gathered}
c_{11}=128, \quad c_{12}=7, \quad c_{13}=6, \quad c_{22}=72, \quad c_{23}=5, \\
c_{33}=32, \quad c_{44}=18, \quad c_{55}=12.25, \quad c_{66}=8 \quad \text { in } \mathrm{MPa}, \\
T_{0}=300 \mathrm{~K}, \quad \rho=2000 \mathrm{~kg} / \mathrm{m}^{3}, \\
K_{1}=100, \quad K_{2}=50, \quad K_{3}=25 \quad \text { in } \mathrm{W} / \mathrm{mK}, \\
\beta_{1}=0.04, \quad \beta_{6}=0.06, \quad \beta_{3}=0.09 \quad \text { in } \mathrm{MPa} / \mathrm{K}, \\
\varepsilon_{1}=0.001 \quad\left(\varepsilon_{1}=\frac{T_{0} \beta_{1}^{2}}{\rho C_{e} c_{11}}\right), \quad \tau_{0}=2.10^{-7} \text { second, }
\end{gathered}
$$




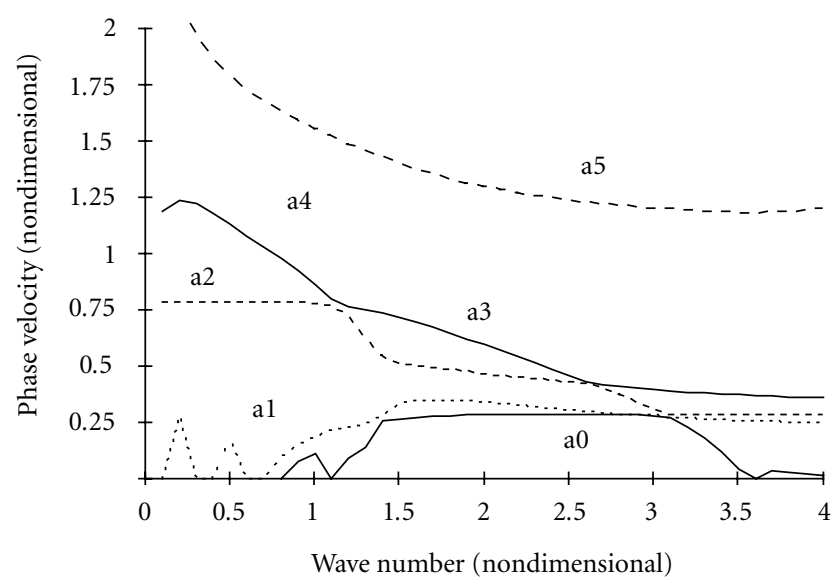

Figure 8.5. Dispersion of the first six flexural modes for $\tau_{0}=2 \cdot 10^{-7}$ second and $\tau_{1}=2 \cdot 10^{-6}$ second.

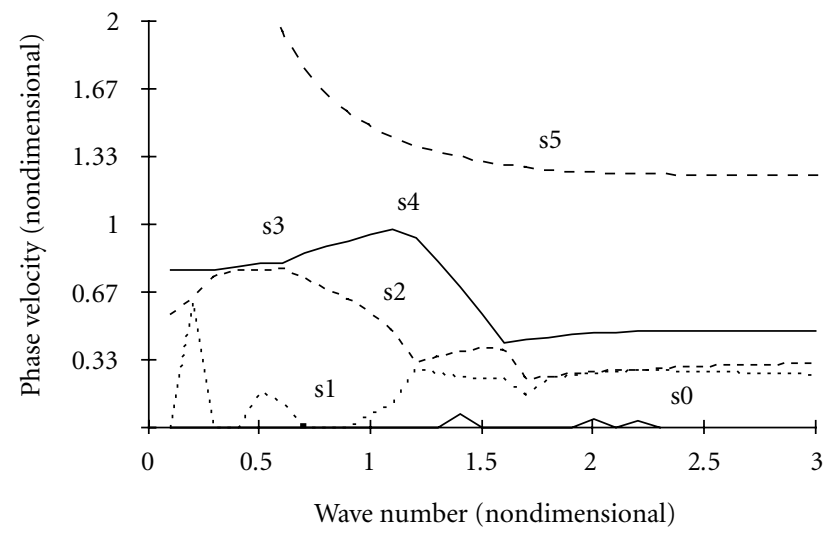

Figure 8.6. Dispersion of the first six extensional modes for $\tau_{0}=2.10^{-7}$ second and $\tau_{1}=2.10^{-6}$ second.

and taking different values of $\tau_{1}$ keeping in mind

$$
\tau_{1} \geq \tau_{0} \geq 0
$$

Dispersion curves in the forms of variations of phase velocity (dimensionless) with wave numbers (dimensionless) are constructed at different values of times, relaxationtime ratios $\left(\tau_{1} / \tau_{0}\right)=2,5,10$, and $\theta=\pi / 2$ for the first six modes of the representative orthotropic plate. Each figure displays three wave speeds corresponding to quasilongitudinal, quasitransverse, and quasithermal at zero wave number limits. It is obvious that the largest value corresponds to the quasilongitudinal mode. Higher modes appear in both cases (flexural and extensional) with $\xi$ increasing. One of these seems to be associated with the rapid change in the slope of the mode. Lower modes (flexural and extensional) are found more influenced by the thermal relaxation times at low values of the wave number. Dispersion curves in Figures 8.1, 8.3, and 8.5 correspond to the flexural wave modes 
(a0, a1, a2, a3, a4, and a5) and Figures 8.2, 8.4, and 8.6 (s0, s1, s2, s3, s4, and s5) correspond to extensional wave modes. The phase velocity of the lowest flexural mode is observed to increase from zero value at zero wave number limits, whereas in the case of the lowest extensional mode, it decreases from a value less than that of the corresponding lowest flexural mode and then tends towards Rayleigh velocity asymptotically with an increase in wave number. The phase velocities of higher modes of propagation, flexural and extensional, attain quite large values at vanishing wave numbers.

Lowest flexural modes ( $\mathrm{a} 0$ ) have nonzero and the lowest extensional modes $(\mathrm{s} 0)$ have zero velocity at vanishing wave numbers, but the phase velocity of these modes also become asymptotically close to the surface wave velocity with increasing value of the wave number. The behavior of higher modes of propagation is observed to be similar to other cases. The effect of thermal relaxation times is observed to be small.

\section{Conclusions}

The interaction of generalized thermoelastic waves with two thermal relaxation times has been investigated for orthotropic media. The horizontally polarized SH wave (3.7) gets decoupled from the rest of the motion and propagates without dispersion or damping, and is not affected by thermal variations on the same plate. The other three waves, namely, quasilongitudinal (QL), quasitransverse (QT), and quasithermal (T-mode), of the medium are found coupled with each other due to the thermal and anisotropic effects. The phase velocity of the waves gets modified due to the thermal and anisotropic effects and is also influenced by the thermal relaxation time. The dispersion characteristics for flexural and extensional waves modes have been taken into consideration. The increasing ratios of thermal relaxation times tend to increase the values of phase velocity of different modes. Within the framework of the generalized theory of thermoelasticity are dispersion curves similar to those of the elastic waves.

\section{References}

[1] I. Abubakar, Free vibrations of a transversely isotropic plate, Quart. J. Mech. Appl. Math. 15 (1962), 129-136.

[2] V. K. Agarwal, On plane waves in generalized thermoelasticity, Acta Mech. 31 (1978), no. 3-4, 185-198.

[3] _ On surface waves in generalized thermoelasticity, J. Elasticity 8 (1978), 171-177.

[4] D. K. Banerjee and Y.-K. Pao, Thermoelastic waves in anisotropy solids, J. Acoust. Soc. Amer. 56 (1974), 1444-1454.

[5] P. Chadwick, Thermoelasticity. The dynamical theory, Progress in Solid Mechanics, Vol. 1 (R. Hill and I. N. Sneddon, eds.), North-Holland Publishing, Amsterdam, 1960, pp. $263-$ 328.

[6] Basic properties of plane harmonic waves in a presented heat conducting elastic material, J. Thermal Stresses 2 (1979), 193-214.

[7] P. Chadwick and L. T. C. Seet, Wave propagation in a transversely isotropic heat-conducting elastic material, Mathematika 17 (1970), 255-274.

[8] D. S. Chandrasekharaiah, Thermoelasticity with second sound: A review, Appl. Mech. Rev. 39 (1986), 355-376.

[9] - Hyperbolic thermoelasticity: A review of recent literature, Appl. Mech. Rev. 51 (1998), 705-729. 
[10] R. S. Dhaliwal and H. H. Sherief, Generalized thermoelasticity for anisotropic media, Quart. Appl. Math. 38 (1980), no. 1, 1-8.

[11] A. E. Green and K. A. Lindsay, Thermoelasticity, J. Elasticity 2 (1972), 1-7.

[12] M. A. Hawwa and A. H. Nayfeh, The general problem of thermoelastic waves in anisotropic periodically laminated composites, Composites Engineering 5 (1995), no. 12, 1499-1517.

[13] F. J. Lockett, Effect of thermal properties of a solid on the velocity of Rayleigh waves, J. Mech. Phys. Solids 7 (1958), 71-75.

[14] H. W. Lord and Y. A. Shulman, A generalized dynamical theory of thermoelasticity, J. Mech. Phys. Solids 15 (1967), 299-309.

[15] C. V. Massalas, Thermoelastic waves in a thin plate, Acta Mech. 65 (1987), 51-62.

[16] C. V. Massalas and V. K. Kalpakidis, Thermoelastic waves in a thin plate with mixed boundary conditions and thermal relaxation, Ingenieur-Archiv 57 (1987), 401-412.

[17] - Thermoelastic waves in a waveguide, Internat. J. Engrg. Sci. 25 (1987), 1207-1218.

[18] A. H. Nayfeh and D. E. Chementi, General problem of elastic wave propagation in multilayered anisotropic media, J. Acoust. Soc. Amer. (1991), 1521-1531.

[19] W. Nowacki, Thermoelasticity, Int. Ser. Monographs in Aeronautics and Astronautics, PWN, Warsaw, 1962.

[20] Dynamic Problems of Thermoelasticity, Noordhoff International Publishing, Leyden, 1975.

[21] P. Puri, Plane waves in generalized thermoelasticity, Internat. J. Engrg. Sci. 11 (1973), 735-744.

[22] , Plane waves in generalized thermoelasticity. Errata, Internat. J. Engrg. Sci. 13 (1975), 339-340.

[23] D. Tao and J. H. Prevost, Relaxation effects on generalized thermoelastic waves, J. Thermal Stresses 7 (1984), 79-89.

[24] K. L. Verma, Thermoelastic vibrations of transversely isotropic plate with thermal relaxations, Internat. J. Solids Structures 38 (2001), 8529-8546.

[25] K. L. Verma and N. Hasebe, On the propagation of generalized thermoelastic vibrations in plates, Engrg. Trans. 47 (1999), no. 3-4, 299-319.

[26] Wave propagation in plates of general anisotropic media in generalized thermoelasticity, Internat. J. Engrg. Sci. 39 (2001), 1739-1763.

[27] Wave propagation in transversely isotropic plates in generalized thermoelasticity, Archive of Applied Mechanics 72 (2002), no. 6-7, 470-482.

K. L. Verma: Department of Mathematics, Government Post Graduate College, Hamirpur, Pradesh 177005, India

E-mail address: klverma@sancharnet.in

Norio Hasebe: Department of Civil Engineering, Nagoya Institute of Technology, Gokio-Cho, Showa-Ku, Nagoya 466, Japan

E-mail address: hasebe@kozo4.ace.nitech.ac.jp 


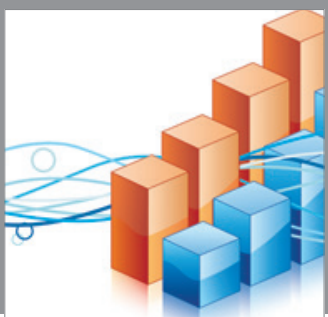

Advances in

Operations Research

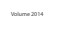

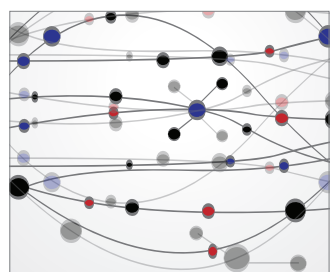

\section{The Scientific} World Journal
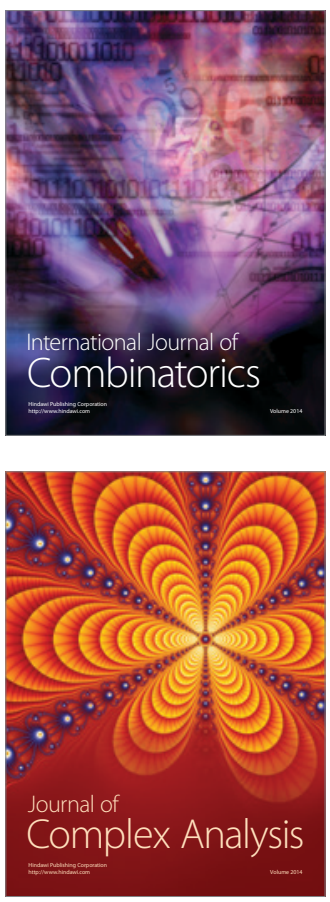

International Journal of

Mathematics and

Mathematical

Sciences
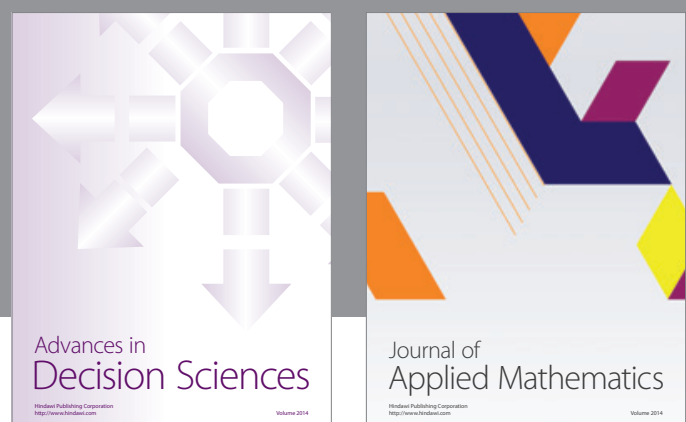

Journal of

Applied Mathematics
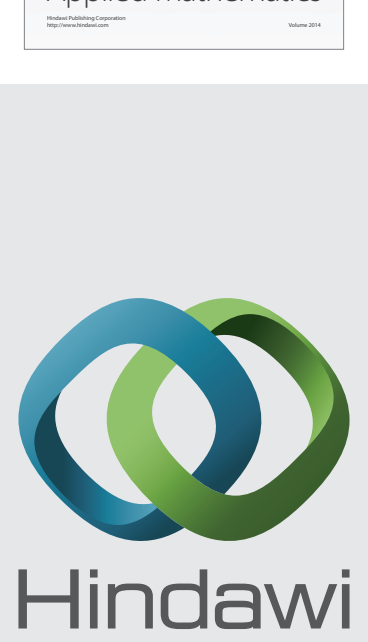

Submit your manuscripts at http://www.hindawi.com
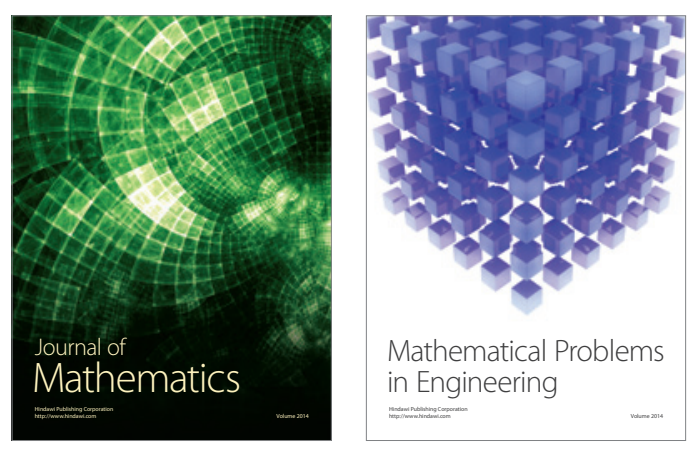

Mathematical Problems in Engineering
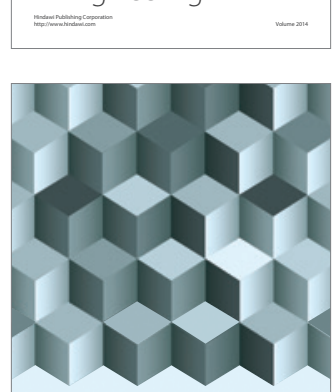

Journal of

Function Spaces
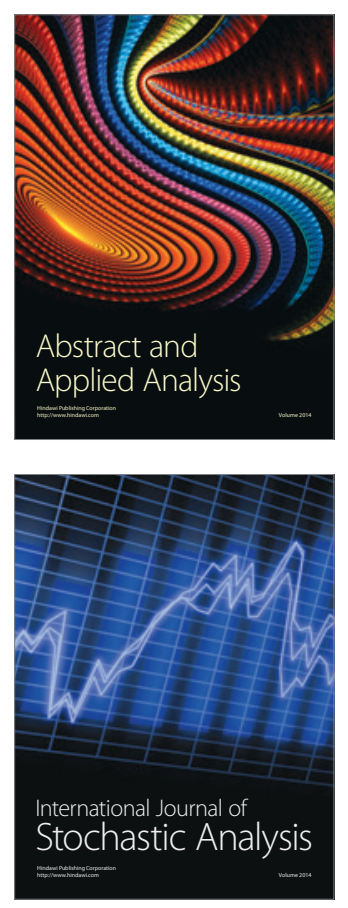

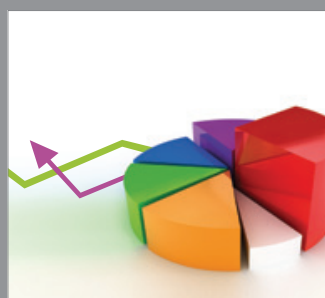

ournal of

Probability and Statistics

Promensencen
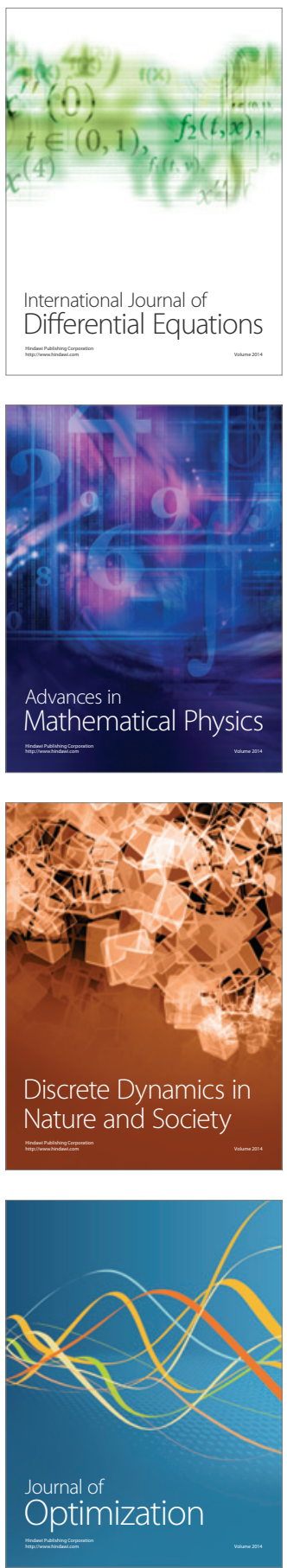